\title{
Échantillons rocheux provenant de « volcans de boue » de Méditerranée orientale
}

\author{
Caroline HUGUEN ${ }^{a *}$, Jean BENKHELIL ${ }^{b}$, Pierre GIRESSE $^{b}$, Jean MASCLE ${ }^{a}$, Carla MULLER, \\ John WOODSIDE ${ }^{c}$, Tiphaine ZITTER ${ }^{c}$, and the Medinaut scientific party \\ ${ }^{a}$ Géosciences-Azur, observatoire océanologique de Villefranche, BP 48, 06235 Villefranche-sur-Mer, France \\ ${ }^{\mathrm{b}}$ LSM, université de Perpignan, avenue de Villeneuve, 66000 Perpignan, France \\ ${ }^{\mathrm{c}}$ Free University, Amsterdam, De Boelelaan, 1085, 1081 HV Amsterdam, the Netherlands
}

Reçu le 24 janvier 2001 ; révisé et accepté le 14 juin 2001

Résumé-En Méditerranée orientale, de nombreuses manifestations argilo-cinétiques, communément appelées «volcans de boue » ont été reconnues et en partie analysées, au cours des vingt dernières années, à travers un grand nombre de méthodes, essentiellement géophysiques. Les résultats de la campagne Medinaut (décembre 1998) permettent une analyse détaillée de ce phénomène grâce à une vingtaine de plongées du submersible Nautile, au sein de deux régions particulièrement riches en phénomènes argilo-cinétiques : le domaine central de la ride méditerranéenne, au sud de la Crète et la région des monts Anaximandre, au sud de la Turquie. Une analyse morphologique, pétrographique, microstructurale et biostratigraphique des clastes prélevés au sein de ces deux régions fournit des indices sur leur évolution. La nature même de ces échantillons, ainsi que leur datation, indiquent un environnement de dépôt marin profond, de type deep-sea-fan distal, au Miocène au centre de la ride méditerranéenne, et un environnement beaucoup plus détritique, probablement de type pente continentale, pour la région des monts Anaximandre. L'examen macroscopique et microstructural de ces échantillons a également permis de mettre en évidence, dans de nombreux cas, un contrôle de leur morphologie par un réseau de microfractures synsédimentaires (veines calcitiques, diaclases) antérieures à leur mise en place sur le fond marin. Enfin, des phénomènes tardifs, tels que des traces d'érosion, des auréoles d'altération et des dépôts pelliculaires calcitiques, ont été observés à la surface de ces clastes et corrélés au contexte géochimique complexe associé à la mise en place des volcans de boue. (C) 2001 Ifremer/CNRS/IRD/Éditions scientifiques et médicales Elsevier SAS

\begin{abstract}
Clasts from 'mud volcanoes' from the eastern Mediterranean. In the eastern Mediterranean, numerous argilo-kinetic manifestations, commonly named 'mud volcanoes', have been identified and studied in some detail during the last twenty years using several techniques. The Medinaut survey (December 1998) has provided new insights into this phenomenon through twenty deep dives performed with the submersible Nautile within two areas particularly active in terms of mud expulsions: the central Mediterranean ridge south of Crete, and the Anaximander mountains area, south of Turkey. The morphology, petrography, microstructure and biostratigraphy of different clasts sampled in the two areas helped to better understand the mud volcano formation and subsequent evolution. Paleoenvironmental conditions, ranging from deep marine deposition in distal deep-sea-fan environment during the Miocene (Mediterranean ridge) to continental margin conditions (Anaximander mountains), have been evaluated for the composition and age of the various rock types. Macroscopic and microscopic structural observations have revealed a close control of clast shapes by sets of early microfractures (calcite veins, joints) created before the clast expulsion to the seabed surface. Surface features, such
\end{abstract}

* Correspondance et tirés à part.

Adresse e-mail : huguen@obs-vlfr.fr (C. HUGUEN).

(C) 2001 Ifremer/CNRS/IRD/Éditions scientifiques et médicales Elsevier SAS. Tous droits réservés

S0399178401011525/FLA 
as erosion marks, alteration aureoles or calcitic coatings, have also been described on most samples. Finally, erosion traces, alteration aureoles and calcitic coating observed for the first time are interpreted as the result of a complex cold seep context related to mud expulsion and associated fluid venting. (c) 2001 Ifremer/CNRS/IRD/Éditions scientifiques et médicales Elsevier SAS

\section{clastes / Méditerranée orientale / plongées profondes / « volcans de boue »}

\section{clasts / eastern Mediterranean / deep dives / 'mud volcanoes'}

\section{INTRODUCTION}

En domaine marin, les phénomènes d'argilo-cinèse aboutissent souvent à la mise en place d'importantes accumulations boueuses sur le fond de la mer, communément appelées « volcans de boue ». Ces derniers sont reconnus dans des contextes géodynamiques variés, parmi lesquels certaines régions de marges passives Vogt et al. 1997 1999: Milkov et al. 1999, 2000) ou, plus fréquemment, les prismes d'accrétion de marges actives (Westbrooke Smith. 1983). Leur origine est liée à la présence, au sein de la colonne sédimentaire sous-jacente, d'un, ou de plusieurs, niveaux d'argiles incomplètement lithifiées ou rechargées en fluides au cours d'épisodes hydrothermaux successifs. La plupart des modèles de mise en place Kopf et al, 1998, 2000) impliquent une relation étroite avec des accidents tectoniques, failles ou chevauchements. Ces derniers servent de conduits à l'ascension des ensembles sous compactés, soit sous l'effet de surpressions engendrées par la surcharge sédimentaire, soit encore du fait de contraintes compressives ou de surproductions de gaz en surpression. D'importants volumes de matériel argileux, au sein desquels sont généralement incorporés de nombreux fragments rocheux (clastes) arrachés aux formations traversées, sont ainsi expulsés sur le fond, associés à d'importantes quantités de fluides, le plus souvent du méthane. Ces ensembles bréchiques, ou «mud breccia» Cita_et_al_ 1996) constituent une caractéristique fréquente des volcans de boue de la Méditerranée orientale, et se matérialisent sur les enregistrements de sonar par une forte réflectivité Volgin e Woodside, 1996.

En Méditerranée orientale, ces structures ont été identifiées pour la première fois vers la fin des années 70, au large de la Crète (Belderson_et_al_, 1974) Par la suite, plusieurs champs d'origine argilo-cinétique ont été reconnus et en partie étudiés dans cette région du prisme méditerranéen Cita_et al. 1998 par outils géophysiques (sonar latéral, sismique réflexion) et carottages au cours des campagnes du programme Training Through Research (TTR), associant l'université de Moscou à divers partenaires Limonov et al. 1996) dans le cadre du Network on Advanced Study Workshops on Mediterranean Marine Geosciences de la Fondation européenne pour la science. Plus récemment, l'usage de sondeurs multifaisceaux a fourni une cartographie d'ensemble de ces structures dans un cadre régional (Mascle et al. 1999. La réalisation de quelques forages courts sur deux de ces volcans dans le sud de la Crète (Rohertson, 1996) a également permis de préciser une partie de leur lithologie, reconnue antérieurement, mais de manière succincte, par carottages (Hsii_et al_ 1978: Cita_et al. 1998; Hiecke et al, 1985: Akhmanov, 1996)

En se fondant sur ces divers travaux de reconnaissance, la campagne Medinaut a été conduite en décembre 1998 avec le submersible de recherches de l'Ifremer le $\mathrm{Nau}$ tile. Cette campagne a permis, lors de vingt plongées, d'effectuer des observations, des prélèvements et des mesures in situ afin d'aborder, de façon pluridisciplinaire, l'analyse de ces manifestations particulièrement actives au sein de deux régions de la Méditerranée orientale (Medinaut/Medineth_shiphoard_scientific parties, 2000) (Gigure - 1) :

- Le domaine central de la ride méditerranéenne (zone Olympie), région où les contraintes compressives résultant de la convergence des plaques européenne et africaine seraient les plus importantes (Mascle et al._1999); - La seconde cible se situait au sud de la Turquie, au sein des monts Anaximandre, région où la mise en place de volcans et de coulées de boue a été décrite dans un contexte associant tectoniques transcurrentes et compressives (Woodside et al. 1998)

La campagne Medinaut a fourni des observations et des échantillons sur plusieurs structures de boue (fgure dont les plus connues sont les volcans Napoli, Milano, Moscou (champ Olympie), Amsterdam, Kula et Kazan (domaine Anaximandre). Ces plongées avaient plusieurs 


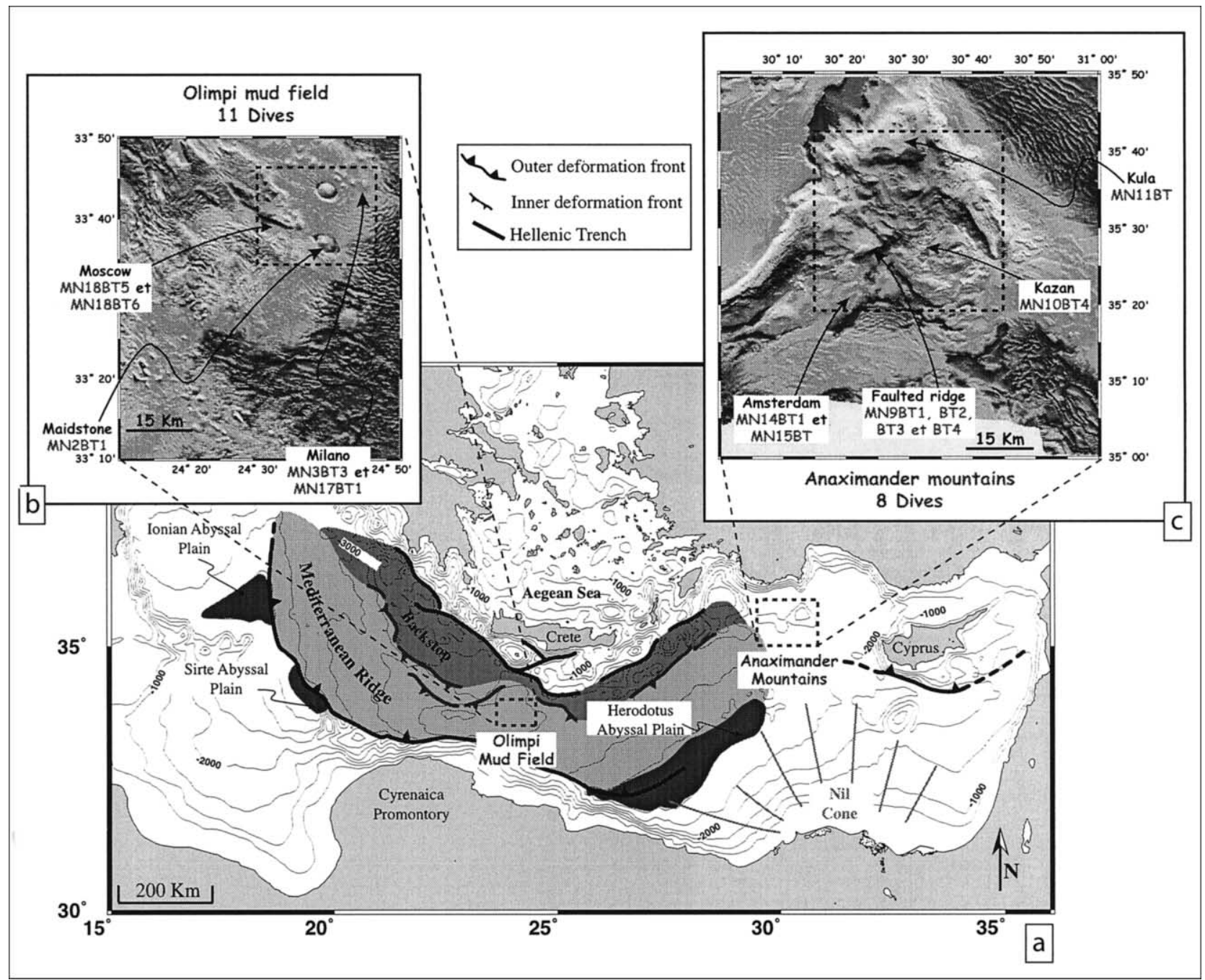

Figure 1. a. Localisation des zones d'étude en Méditerranée orientale (d'après Chaumillon et Mascle, 1996 ; et Robertson et al., 1996). Une localisation plus précise des structures argilo-cinétiques étudiées durant la campagne Medinaut (décembre 1998) est fournie sur fond bathymétrique ombré en b (campagne Prismed 2, 1998) et c (campagne Anaxiprobe, 1995).

Figure 1. a. Location map of the studied areas in the eastern Mediterranean (from Chaumillon and Mascle, 1996 ; and Robertson et al., 1996). Position of argilo-kinetic structures investigated during the Medinaut cruise (December 1998) is given on shaded bathymetry in b (from Prismed 2 cruise, 1998) and c (Anaxiprobe cruise, 1995).

objectifs : (1) la reconnaissance détaillée et la comparaison de plusieurs édifices et de leurs coulées boueuses successives (et phénomènes associés) ; (2) l'observation de leurs éventuels liens génétiques avec des accidents tectoniques; (3) la recherche et l'évaluation d'éventuels panaches de gaz, phénomènes de suintement, lacs de saumures et hydrates de gaz, ainsi que l'impact de ces phénomènes sur la physico-chimie de la colonne d'eau et des sédiments superficiels ; et (4) l'observation et l'ana- lyse des écosystèmes microbiologiques et benthiques associés à l'émanation de fluides et de gaz ainsi que celles des phénomènes biogéochimiques qui en sont issus.

Cet article concerne plus spécifiquement une étude conduite sur une partie des clastes prélevés au sein de ces deux régions. Il se fonde plus précisément sur une analyse morphologique, pétrographique et microstructurale de dix-neuf échantillons prélevés dans les coulées boueuses 
issues des volcans Maidstone, Milano et Moscou (champ Olympie) et des structures Amsterdam, Kazan et Kula, ainsi que sur la «faulted ridge », escarpement tectonique de dimensions importantes (région Anaximandre) (6gure d).

Ce travail avait pour but principal la recherche d'indices de déformation et de minéralogenèse enregistrés par ces roches et issus des conditions spécifiques (tectoniques et géochimiques) de leur mise en place dans le volcan de boue. L'analyse de ces indices devait permettre en retour d'évaluer les différentes contraintes auxquelles ces clastes ont été soumis, avant, pendant et après leur remontée et leur mise en place sur le fond de la mer. Les recristallisations minérales ont été l'objet d'une analyse par diffractométrie aux rayons $\mathrm{X}(\mathrm{RX})$, et d'une observation au microscope électronique à balayage (MEB) couplée à une étude par microsonde Tracor, afin de déterminer les éventuelles variations d'environnement thermique ou chimique (épisodes hydrothermaux, influence de la crise salifère messinienne), auxquelles ces roches ont été soumises au cours de leur histoire. Dans la mesure du possible, ces informations ont été complétées par des datations biostratigraphiques.

\section{CADRE STRUCTURAL DU VOLCANISME BOUEUX}

En Méditerranée orientale, l'environnement tectonique global résulte de diverses interactions entre le déplacement relatif vers le Nord (par rapport à l'Europe) de la plaque Afrique et celui, plus récent et assimilable à une rotation anti-horaire par rapport à l'Europe, de la microplaque anatolienne Le Pichon et al. 1995: Reillinger ed al. 1997; Mc Clusky et al, 2000). Au sud de la Crète, la subduction hellénique, frontière entre ces deux plaques, est soulignée par un vaste prisme d'accrétion, la ride méditerranéenne, édifice de dimensions considérables (1 $500 \mathrm{~km}$ de long pour 150 à $300 \mathrm{~km}$ de large), caractérisé par une intense déformation de surface et d'importantes expulsions de boue Cita et al. 1996; Limonov ed al. 1996) Le relais vers l'arc de Chypre et, au delà, avec la collision dans l'est de la Turquie (Woodside et_al 1998) s'effectue par une zone de déformation complexe qui passe par un ensemble de reliefs et de bassins incluant la fosse de Rhodes, les monts Anaximandre et la ride de Florence.
Les manifestations boueuses les plus importantes s'expriment particulièrement bien au sein du domaine central de la ride méditerranéenne, région où cet édifice est à la fois le plus étroit, le plus élevé et où se produit, peut-être, un début de collision continentale entre les marges africaine et égéenne (Mascle et al._1999). Dans un tel contexte plusieurs facteurs semblent favorables à la mise en place de volcans de boue, tels que par exemple les effets de la compression régionale, ceux de la surpression au sein des niveaux de boue liés à l'épaisseur considérable des séries sédimentaires susjacentes (de l'ordre de $12 \mathrm{~km}$ de sédiments selon De Voogd et al. 1992) la présence de nombreux accidents tectoniques affectant l'ensemble de la ride méditerranéenne et facilitant ainsi l'échappement des fluides vers la surface CChaumillon et Mascle, 1997; Mascle et al. 1999). Souvent considérés comme une barrière étanche aux remontées de fluides et de boues (Limonov et al, 1996) les dépôts évaporitiques messiniens semblent vraisemblablement d'épaisseur trop faible dans cette région, pour être a l'origine de surpressions importantes. C'est dans cet environnement spécifique que s'observe la plupart des volcans de boue, en particulier au long de la bordure septentrionale du prisme, à proximité de son butoir continental, la marge crétoise qu'il chevauche Kopf et al, 2000).

Le champ Olympie, situé au sud de la Crète et caractérisé par des structures boueuses de dimensions variables (entre 1 et $15 \mathrm{~km}$ de diamètre et de 100 à $200 \mathrm{~m}$ d'élévation) est, à l'heure actuelle, le plus étudié et le mieux connu de ces « champs de boue ». Parmi l'ensemble des structures boueuses qui le composent, seules trois d'entre elles, offrant des morphologies assez différenciées, sont abordées ici (figure - 1 : (1) la structure Moscou, qui se matérialise par une ride de plus de $8 \mathrm{~km}$ de long, est clairement mise en place au droit d'une zone de failles transcurrentes actives Huguen et al. soumis; (2) le volcan de boue Maidstone, de forme elliptique et entouré d'une dépression profonde (environ $100 \mathrm{~m}$ ), se situe, quant à lui, dans le prolongement de l'accident tectonique contrôlant le volcan Moscou, et apparaît également contrôlé par cette fracture; (3) enfin, le volcan Milano, de forme subcirculaire et de dimensions plus réduites, se caractérise par d'importantes coulées très réflectives sur l'imagerie sonar et par un contrôle tectonique moins net.

Au sud de la Turquie, entre l'extrémité orientale de la ride méditerranéenne et la ride de Florence, les monts Anaximandre (fgure - ل ) correspondent quant à eux à un 
ensemble de reliefs tectoniquement détachés de la Turquie méridionale Woodside et al. 1998) Ces reliefs sont actuellement soumis à une déformation complexe, associant accidents compressifs et transcurrents et leur domaine oriental est, vers l'Est, en continuation avec la ride de Florence, large zone à valeur décrochante correspondant à la terminaison de la branche nord-occidentale de l'arc de Chypre. Dans cette région, à l'instar de la ride méditerranéenne, la mise en place des structures boueuses est facilitée par la présence d'accidents tectoniques importants ainsi que par l'absence de dépôts évaporitiques messiniens (Woodside et al. 1998). La majeure partie des épanchements boueux y est de forme sub-circulaire, de diamètres variant entre 1 et $5 \mathrm{~km}$, pour une centaine de mètres de hauteur (Woodside_et_al, 1998) Au cours de la campagne Medinaut, plusieurs plongées ont été effectuées sur le volcan Amsterdam ( $5 \mathrm{~km}$ de diamètre), localisé sur un réseau de failles actives et offrant à la fois une morphologie très plane et de nombreuses coulées. Trois plongées ont été effectuées sur les volcans Kula, sur lequel des hydrates de gaz avaient préalablement été prélevés (Woodside et_al, 1998) et Kazan, l'un et l'autre également situés à proximité d'accidents tectoniques majeurs. Enfin, une plongée a été dédiée à l'étude d'un grand escarpement de faille de plus de $500 \mathrm{~m}$ de rejet vertical, le long duquel ont été observés de nombreux effluents gazeux («faulted ridge »).

\section{RÉSULTATS}

\subsection{Pétrographie, microstructure et biostratigraphie des clastes}

\subsubsection{Champs de boue Olympie}

Cinq clastes, provenant de quatre plongées sur les volcans Maidstone (MN2), Milano (MN3 et MN17) et Moscou (MN18), ont été étudiés (figure des grès plus ou moins calcaires, dont la granulométrie varie du grain moyen hétérométrique (composé de quartz émoussés, feldspaths altérés et débris coquilliers - MN17 BT1, MN3BT3a) au grain fin homogène (MN2BT1, MN18BT6). Des clastes à dominante marneuse ont été récoltés sur les volcans Milano (MN3BT3b, fogure $2 B$ ]) et Moscou (MN18BT6,), montrant une stratification peu marquée, parfois soulignée par des lentilles de calcite de taille centimétrique (Ggure 2B33). Les clastes gréseux sont caractérisés par une morphologie assez massive, à faces planes plus ou moins émoussées (MN2BT1, MN7BT1, MNT18BT5), résultant de l'association de structures sédimentaires et de fractures plus ou moins prononcées. $\mathrm{Au}$ sein des clastes marneux, les structures sont bien exprimées par un réseau de diaclases (MN3BT3b) ou de filons calcitiques (MN18BT6) ; ces derniers sont parfois dégagés et tapissent certaines faces de l'échantillon (MN18BT5). Le diffractogramme RX de ce matériel révèle la présence de calcite, d'aragonite, de quartz et d'anhydrite. La plupart des roches étudiées sont partiellement enrobées par une auréole ferrugineuse (MN2BT1, MN3BT3a), particulièrement développée au niveau des clastes à dominante gréseuse des volcans Moscou (MN18BT5) et Milano (MN17BT1). Certains échantillons ont livré des nannofossiles datés du Miocène inférieur (MN18BT5, zone NN3 pour Moscou ; MN3BT3, zone NN4 pour Milano), ou du Miocène moyen (MN3BT3, zone NN6 pour Milano ; MN2BT1, zone NN5 pour Maidstone).

\subsubsection{Domaine Anaximandre}

Quatre structures («faulted ridge », MN19; Kazan, MN10 ; Kula, MN11 ; Amsterdam, MN14/15) ont fourni les vingt échantillons analysés. La lithologie y est également à dominante gréseuse, avec des proportions variables de carbonate; localement, des marnes sombres ont été prélevées («faulted ridge», MN9BT3/4). Les grès généralement fins, souvent perturbés par des structures sédimentaires (slumps, MN14BT4 (Ggure 2D) et stratifications obliques, MN14BT1), montrent une stratification généralement soulignée par des lamines argileuses plus ou moins continues. La forme des clastes est variable, allant de plaques peu épaisses à bords émoussés et surfaces accidentées de sillons (Kula, Ggure 2Aل ) jusqu'à des blocs massifs à faces planes (Amsterdam). Un échantillon de forme allongée, prélevé sur le volcan Kazan (MN10BT4), est constitué de grès fin riche en microstructures sédimentaires (slumps, microfailles synsédimentaires). Enfin, l'ensemble des échantillons provenant de la «faulted ridge » est très hétérogène d'un point de vue morphologique. D'une manière générale, la forme des clastes est très souvent contrôlée par les deux surfaces représentées par la stratification, formant généralement les faces de blocs, et un réseau de diaclases et de filons minéralisés perpendiculaires à la stratification (figure 2B3]). Ce dispositif façonne des blocs losangiques, particulièrement nets dans le cas d'Amsterdam (figure 2D), ou aboutit à des formes très hétérogènes comme dans le cas de Kula et Kazan. Lorsque les filons sont d'épaisseur 


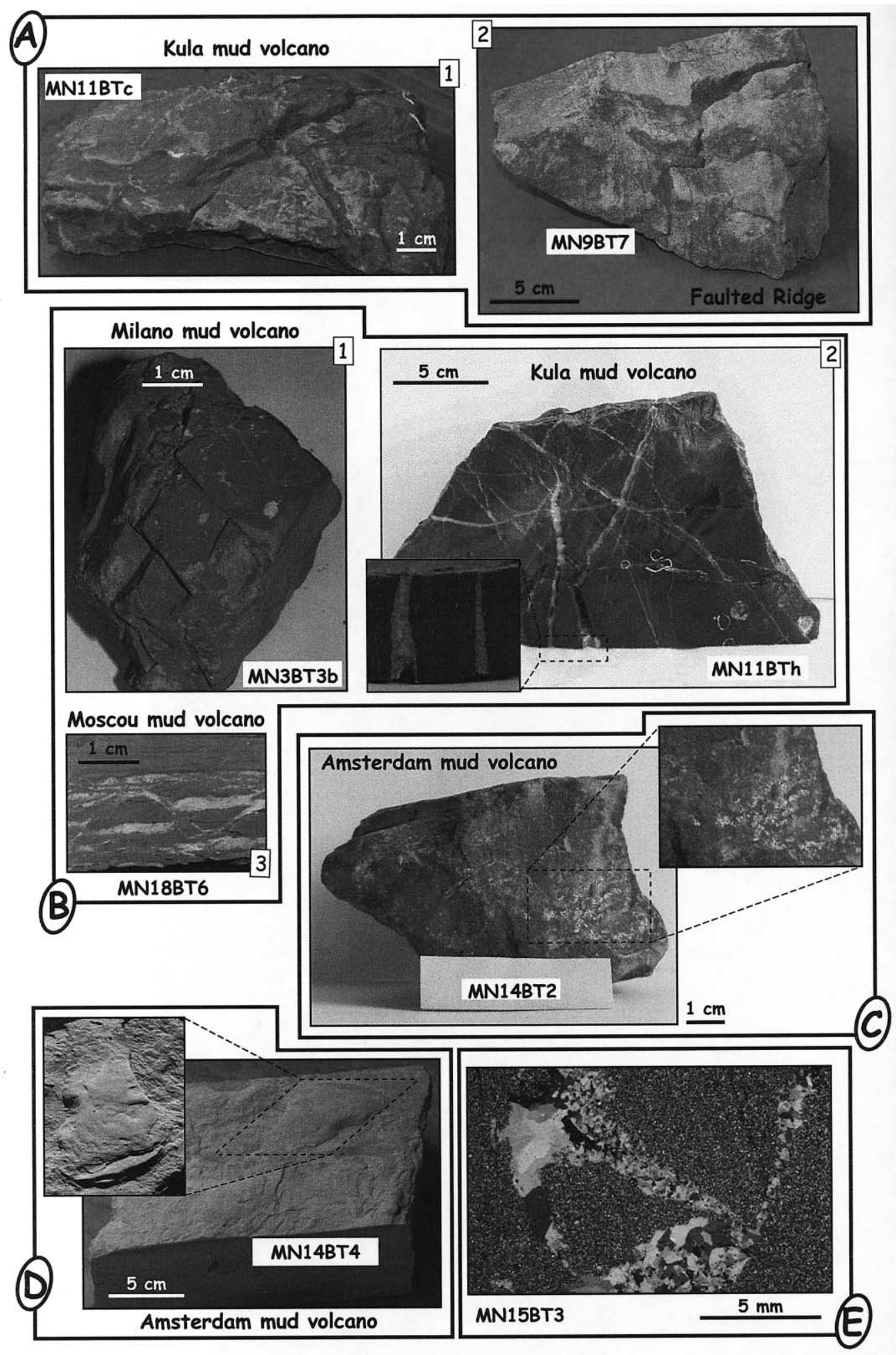


significative, ils peuvent avoir été préférentiellement altérés conduisant à la formation de sillons, parfois profonds de quelques millimètres, visibles à la surface du claste (MN10BT4, MN11BTc/g). En lame mince figure $\mathrm{2E}$ ), ces remplissages montrent des épontes très irrégulières avec, localement, des grains de matrice inclus dans le remplissage calcitique (MN11BTe, MN15BT4, MN15BT3, ...) ; cette observation implique une mise en place dans un matériel incomplètement lithifié, ou rechargé en fluides au cours d'un épisode hydrothermal. L'ensemble des clastes échantillonnés montre des auréoles d'altération ferrugineuse sur une ou plusieurs des faces. Des concrétions centimétriques d'oxydes, de couleur rouille, ont également été identifiées par rayons X sur l'échantillon MN10BT4 (Kazan); elles sont associées à une argile d'altération orangée en remplissage de cavités. Outre les traces d'altération ferrugineuse, la surface de certains échantillons, en particulier ceux provenant d'Amsterdam, offre une patine lustrée (MN14BT2/9 et MN15BT3), quelquefois affectée de stries millimétriques (MN14BT2, fgore 2d). Cette patine observée sur les faces non oxydées du claste peut, dans certains cas (MN14BT2), recouvrir la quasi-totalité de l'échantillon. Comme au sein de la zone Olympie, des âges du Miocène moyen (Langhien à Serravalien) ont été obtenus à partir des nannofossiles contenus dans les échantillons de la «faulted ridge » ( zone NN6) et du volcan Amsterdam (zone NN5/6). En revanche, les clastes prélevés sur Kazan et Kula indiquent, pour la plupart, un âge crétacé supérieur (Campanien supérieur et Maastrichtien).

\subsection{Dépôts pelliculaires observés à la surface des clastes}

En majorité, les échantillons prélevés sur le volcan Amsterdam sont caractérisés par la présence de dépôts pelliculaires en surface, qui ont été analysés par diffractométrie aux RX et observation au MEB couplé à la microsonde Tracor sur les échantillons MN14BT2 et MN14BT9.

Cette pellicule superficielle (fgure $3 d$ ), généralement anisopaque mais toujours infra-millimétrique, est composée de sparite calcitique (figure 3 b). L'analyse diffractométrique, confirmée par les mesures à la microsonde, indique dans la majorité des cas une calcite fortement magnésienne, avec un pourcentage molaire de magnésium de l'ordre de $30 \%$. Toutefois, l'enduit de MN14BT9 montre plusieurs plages de calcite faiblement magnésienne et même de calcite pure. Dans cet enduit, la présence de chlorure de sodium, inclus ou recouvert par la calcite, est également décelable à plusieurs reprises ; plus rarement, une série de petits cristaux tabulaires de gypse à été observée en surface (figure $3 d$.).

En Méditerranée orientale, de nombreux phénomènes géochimiques secondaires sont observés en association avec les volcans de boue. L'ascension de saumures chaudes issues des couches infra-salifères à par exemple été décrite au sein de la zone Olympie, et la présence d'hydrates de gaz (clathrates), observée sur de nombreux volcans tels que Kula (Woodside et al 1998 ou, plus récemment, Amsterdam lHaese et De Lange, 2000. Medinauth/Medinet, 2000). La présence de ces cristaux d'hydrates de gaz a également été supposée sur la structure Milano (zone Olympie) sur la base de critères géochimiques De_Lange_et Brumsack, 1998a, 1998b)

Dans un tel contexte, caractérisé par des concentrations de méthane et de soufre de 1000 à 2000 fois supérieures aux valeurs habituellement mesurées dans la colonne d'eau Haese et De Lange, 2000: Medinauth/Medinet. 2000, carbonates, gypse et chlorure de sodium peuvent, suivant les cas, précipiter séparément ou en association

Figure 2. Photographies des échantillons les plus caractéristiques prélevés par le Nautile au cours de la campagne Medinaut. A. Exemples typiques d'érosion différentielle des veines calcitiques. B. Exemples de préfracturation des clastes par un réseau de diaclases (Milano) ou de veines de calcite (Kula), contrôlant la géométrie des échantillons. C. Illustration caractéristique des dépôts pelliculaires de calcite observés à la surface de certains clastes, en particulier dans la région des monts Anaximandre. D. Exemple de déformation synsédimentaire (micro-slump) à la surface d'un claste massif et de forme géométrique prélevé sur Amsterdam. E. Photographie du remplissage des filons observé au microscope polarisant, illustrant clairement la présence de deux générations de cristaux de calcite de tailles différentes. Les épontes irrégulières du filon ainsi que les éléments de matrice englobés dans le remplissage indiquent clairement une mise en place synlithification.

Figure 2. Photographs of the most characteristics clasts sampled by submersible during the Medinaut cruise. A. Typical examples of selective erosion along calcite veins. B. Examples of clasts pre-fractured by diaclasis (Milano) or calcite veins (Kula) networks, closely involved in their morphologies. C. Illustration of the thin calcitic deposits described at the surface of some samples, especially in the Anaximander mountains area. D. Typical synsedimentary deformation (micro-slump) observed at the surface of a massive geometric clast sampled on Amsterdam mud volcano. E. Microphotograph of the filled veins observed in thin-sections and clearly composed of two distinct calcite generations of different sizes. Their quite irregular boundaries as well as the matrix particles enclosed indicate a synlithification emplacement. 

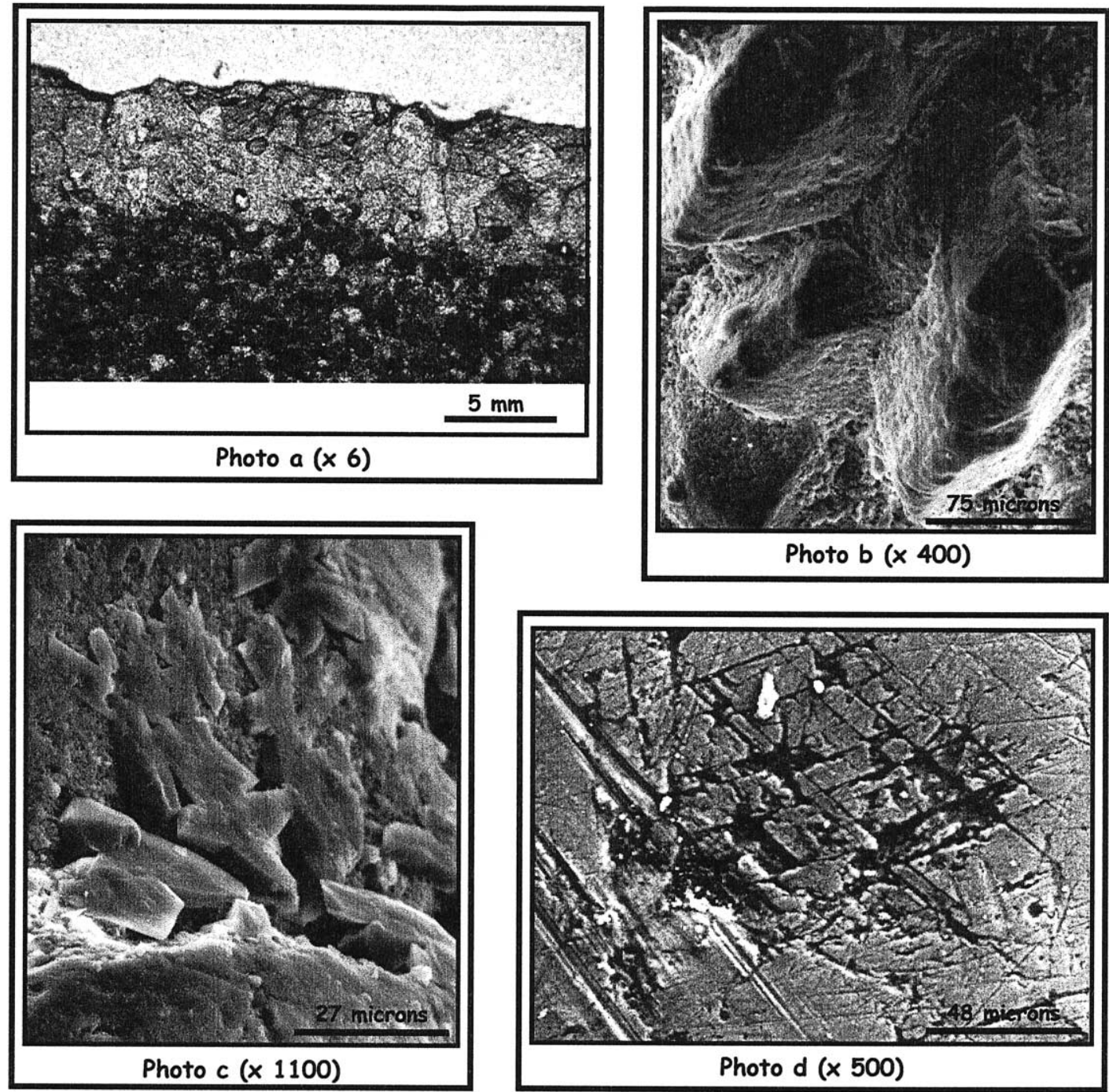

Figure 3. Illustrations des dépôts pelliculaires et traces d'érosion observés à la surface des clastes. a. Lame mince illustrant une pellicule calcitique superficielle infra-millimétrique, partiellement dissoute en plusieurs endroits, en association avec l'apparition de concentrations brunes d'oxydes de Fe et Mn proches de la surface. b. Photographie au MEB de cristaux rhomboédriques typiques de la calcite, observés sur une grande partie de la surface des échantillons analysés. c. Cristaux tabulaires de gypse observés en plaquage diffus sur la pellicule calcitique. d. Observation en plan au MEB de figures de dissolution de la calcite, qui suivent le réseau rhomboédrique du minéral. Les directions sécantes observées dans la partie gauche de la photo sont interprétées comme des stries d'origine tectonique.

Figure 3. Illustrations of surface deposits and erosion. a. Thin section of an inframillimetric calcitic coating partly dissolved in some places and associated with brown concentrations of $\mathrm{Fe}$ and $\mathrm{Mn}$ oxides close to the surface. b. SEM photograph of typical rhombohedric calcite crystals, observed on most of the surface. c. Tabular gypsum crystals locally observed above the surface calcitic coating. d. SEM observation of selective calcite dissolution, along the rhombohedric crystal lattice. Oblique lineations observed on the left part of the photograph corresponds to tectonic striations.

sur les clastes; des variations importantes dans la nature et l'association de ces précipitations étant observées d'un site à un autre. Dans cette étude, le dépôt de calcite magnésienne apparaît plutôt comme une conséquence de la sursaturation des eaux dans cette région de la Méditerranée orientale, où la formation de ce minéral est communément observée Milliman et Müller 1973. Milliman, 1974). La précipitation de calcite pure a déjà 
été décrite Vengosh et al._1998 en association vraisemblable avec une dessalure des eaux interstitielles du sommet de la colonne sédimentaire, elle même induite par la déstabilisation des hydrates de gaz. Nous pensons que le dépôt de calcite pure, localement observé sur certains clastes du volcan de boue Amsterdam pourrait procéder d'un mécanisme identique.

A plusieurs reprises, des stries ont été observées à la surface de ces enduits (figure $3 d$ ), et interprétées comme des figures de dissolution des cristaux calcitiques qui soulignent, en relief négatif, le réseau rhomboédrique du minéral. Ces indices de dissolution sont confirmés par l'observation en lame mince, qui montre localement la disparition complète de la pellicule de calcite sparitique, associée à l'apparition de concentrations brunes caractérisées par la présence de fer $(2-3 \%)$ ou de manganèse $(<1 \%)$ d'après les analyses à la microsonde. Localement, de la goethite a pu être mise en évidence, ainsi que des amas de kaolinite très distincts des argiles micacées que l'on trouve majoritairement dans les cortèges argileux des clastes (Rohertson et Kopf, 1998) Ce phénomène de dissolution de la calcite est le résultat d'une percolation d'eaux agressives postérieure à l'enrobage des clastes, probablement également impliquée dans les processus secondaires d'oxydation, voire d'altération des minéraux argileux. Par endroits, on remarque sur l'enduit calcitique la superposition d'un second ensemble de stries, de directions différentes de celles contrôlées par la dissolution du réseau calcitique et également plus profondes. Ces stries pourraient résulter de contraintes mécaniques subis par les clastes pendant leur ascension le long des plans de faille.

\section{DISCUSSION}

Les volcans de boue constituent une opportunité exceptionnelle d'échantillonnage des formations sédimentaires profondes, habituellement uniquement accessibles par forages. En effet, ces structures permettent la remontée sur le fond, de nombreux fragments rocheux correspondant à des sédiments anciens, déposés voici plusieurs millions d'années et enfouis à l'heure actuelle sous d'épaisses formations sédimentaires.

Bien que basé sur un nombre relativement restreint de prélèvements, en raison du caractère pluridisciplinaire de la campagne et des outils de prélèvement disponibles, ce travail aboutit a plusieurs conclusions concernant diver- ses étapes de l'histoire de ces échantillons, depuis leur environnement de dépôt et leur structuration synsédimentaire, jusqu'à leur évolution tardive, postérieure à leur mise en place au sein du volcan de boue.

La lithologie et l'âge de ces échantillons permettent de préciser certains aspects concernant les conditions de dépôt des sédiments qui les constituent. Au niveau de la ride méditerranéenne, plusieurs études comparables ont déjà été conduites mettant en évidence quatre types lithologiques principaux : calcaires (35\% des clastes), grès $(10 \%)$, siltstones $(5 \%)$ et argiles $(50 \%)$, parmi plus de 300 échantillons étudiés CAkhmanov, 1996; Akhmahov et al, 2000 échantillons, d'âge variant entre le Crétacé supérieur et l'Éocène terminal, à prédominance calcaire et argileuse, indiquent le plus souvent un dépôt en domaine marin profond, caractérisé par une sédimentation hémipélagique et biogénique, avec un apport épisodique de matériel terrigène par des courants de turbidité Cakhmanov_1996.

Les échantillons de la campagne Medinaut provenant de la zone Olympie, composés essentiellement de grès plus ou moins calcaires alternant avec des clastes à dominante marneuse, confirment ces observations antérieures, ainsi que l'interprétation qui en a été déduite en terme de paléoenvironnement de dépôt CAkhmanov. 1996: Akhmanov et al. 2000). De plus, les âges Miocène inférieur (volcans Moscou et Milano) à Miocène moyen (volcan Maidstone) obtenus sont en bon accord avec ces résultats ; il est donc probable qu'un environnement marin profond de type deep-sea-fan distal, associé à un prisme d'accrétion probablement encore très précoce, prédominait au Miocène au sein de la zone Olympie.

Les échantillons provenant des monts Anaximandre sont, quant à eux, caractérisés par des faciès à dominante gréseuse contenant des proportions de carbonate inférieures à celles observées au niveau de la RM. De même, les clastes marneux sont beaucoup moins abondants et proviennent pour la plupart de structures tectoniques telles que la «faulted ridge ». L'influence détritique apparaît donc beaucoup plus prononcée sur ces échantillons, ce qui se conçoit aisément sachant que les monts Anaximandre sont des reliefs détachés de la marge turque (Woodside et al. 1998). Au moment de leur sédimentation, ces roches se situaient donc vraisemblablement plutôt dans un contexte de pente continentale. Les âges obtenus dans cette région sont en général beaucoup plus anciens, certains des clastes sont en effet datés du Campanien 
(Kula) et du Maastrichtien (Kazan). Pour le volcan Amsterdam, situé plus à l'Ouest (figure - les âges sont comparables à ceux observés pour les structures de la zone Olympie, avec une dominance sectorielle d'un âge Miocène moyen (zones NN5 et NN6). Ces roches, de composition carbonatée dominante avec alternance de lits argileux, semblent ainsi indiquer pour le Miocène inférieur un environnement de dépôt marin plus profond, plus proche de celui déduit au niveau de la zone Olympie. L'abondance d'indices de déformation synsédimentaire (slumps, microfailles) observables sur ces échantillons témoigne cependant également d'instabilités sédimentaires probablement liées à une activité tectonique importante à l'échelle régionale, au moment du dépôt de ces sédiments, ou dans un stade précoce de leur lithification.

Outre ces aspects paléoenvironnementaux, l'examen macroscopique et microstructural des clastes a révélé, dans de nombreux cas, des morphologies clairement contrôlées par des réseaux de microfractures (veines et diaclases). Cette observation a conduit à l'hypothèse d'un contrôle tectonique de la morphologie des blocs rocheux, antérieur à leur mise en place au sein des volcans de boue.

Les réseaux de diaclases (MN3BT3b, figure 2Bd]) et veines calcitiques (MN11BTh, fgure 2B2), parfois clairement conjugués, ont été observés à plusieurs reprises sur ces échantillons. De plus dans certains cas, ces microfractures présentent des orientations parallèles aux faces des blocs aboutissant à une morphologie caractéristique, très souvent observée pour les échantillons des volcans Milano, Moscou, Kula et Amsterdam (foure 2). Cette observation, confortée par la présence de nombreux indices de mise en place synsédimentaire (épontes onduleuses, particules d'encaissant englobées dans le remplissage), semble indiquer que ces filons se seraient mis en place dans un stade précoce de lithification des niveaux rocheux à l'origine des clastes (Maltman, 1994) peu après leur sédimentation, et en liaison avec des circulations hydrothermales. Ainsi, sous l'effet de la surpression provoquée par les argiles sous-compactées sous-jacentes, les formations sédimentaires indurées auraient été préférentiellement fracturées le long de ces veines, aboutissant dans certains cas à la présence de filons dégagés qui tapissent les faces de certains des clastes. On remarque toutefois une influence importante de la lithologie des échantillons sur la présence, ou non, de ce type de microstructures. En effet, ces dernières sont généralement beaucoup plus développées au sein des échantillons gréseux à composante carbonatée importante où les phénomènes de pression-dissolution-recristallisation sont favorisés (Maltman, 1994) ; les clastes à dominance marneuse, ou affectés de nombreux lits argileux, offrent quand à eux des formes beaucoup plus irrégulières, souvent contrôlées par les seules surfaces de stratification. Au vu de ces observations, on peut donc conclure en ce qui concerne la forme des clastes à une influence combinée de la lithologie (présence de lits argileux dans la stratification) et de la préfracturation par un réseau de microfractures (veines ou diaclases).

L'analyse détaillée de ces échantillons a enfin permis de proposer quelques hypothèses concernant l'évolution tardive des clastes. Une fois remontés à la surface par les coulées boueuses, ces clastes vont être soumis à un environnement géochimique agressif lié aux nombreuses circulations de fluides et de gaz qui accompagnent le phénomène de volcanisme boueux. Leur morphologie va donc être légèrement modifiée par le biais d'un certain nombre de phénomènes secondaires de dissolutions et précipitations, peu connus jusqu'à présent et qui donnent à l'échantillon un aspect « érodé » caractéristique (Ggure 2A). Le caractère morphologique le plus étonnant résultant de ces phénomènes réside dans la présence de sillons profonds de plusieurs centimètres à la surface du claste. Lors d'une observation préliminaire, ces structures peuvent être interprétées comme les traces d'une altération subaérienne de ces blocs ; une analyse plus poussée et en particulier une observation en section de ces blocs montrent cependant que ces sillons correspondent la plupart du temps à une érosion différentielle des veines présentes dans le claste. Ces filons majoritairement calcitiques sont en effet beaucoup plus sensibles à l'environnement hydrothermal acide, et sont donc dissous préférentiellement. Associés à ces phénomènes "d'érosion sous marine », des précipitations d'oxydes de fer et manganèse, ainsi que des kaolinites, produit d'altération des micas ont été analysées au niveau d'auréoles brunes, très souvent observées sur les faces des clastes en contact avec la colonne d'eau. Certains échantillons, en particulier ceux provenant du volcan Amsterdam, sont recouverts de dépôts calcitiques pelliculaires liés à l'ascension de saumures chaudes au niveau des volcans de boue. De nombreux indices de dissolution partielle de cette pellicule ont été observés et interprétés en liaison avec une percolation tardive de fluides hydrothermaux acides, très probablement également responsables des phénomènes d'oxydation et d'altération observés sur la plupart des clastes. 


\section{RÉFÉRENCES}

Akhmanov, G., 1996. Lithology of mud breccia clasts from the Mediterranean Ridge. Mar. Geol. 132, 151-164.

Akhmanov, G., Ivanov, M.K., Woodside, J.M., Cita, M.B., Kenyon, N.H., 22-25 November 2000. Evolution of sedimentation patterns on the north African margins: an update from studies of recent mud volcanic deposits. CIESM Workshop, “African continental margins of the Mediterranean sea”, Djerba, Tunisia.

Belderson, R.H., Kenyon, N.H., Stride, A.H., 1974. Calabrian Ridge, a newly discovered branch of the Mediterranean Ridge. Nature 247, 453-454.

Chaumillon, E., Mascle, J., 1997. From foreland to forearc domains: New multichannel seismic reflection survey of the Mediterranean Ridge accretionary complex (Eastern Mediterranean). Mar. Geol. 138, 237-259.

Cita, M.-B., Erba, E., Lucchi, R., Pott, M., van der Meer, R., Nieto, L., 1996. Stratigraphy and sedimentation in the Mediterranean Ridge diapiric belt. Mar. Geol. 132, 131-150.

Cita, M.B., Camerlenghi, A., Erba, E., McCoy, F.W., Castradory, D., Cazzani, A., Guasty, G., Gianbastiani, M., Lucchi, R., Nolli, V., Pezzi, G., Redaelli, M., Rizzi, E., Torricelli, S., Violanti, D., 1998. Discovery of mud diapirism on the Mediterranean Ridge. A preliminary report. Boll. Soc. Geol. It. 108, 537-543.

De Lange, G.-J., Brumsack, H.-J., 1998a. Pore water indication for the occurrence of gas hydrates in Eastern Med dome structures, ODP Scientific Results, vol. 160, pp. 569-574.

De Lange, G.-J., Brumsack, H.-J., 1998b. The occurrence of gas hydrates in Eastern Med mud dome structures as indicated by pore water composition. In : Henriet, Mienert (Eds.), Gas Hydrates: Relevance to a World Margin Stability and Climatic Change. Geological Society, Special Publication 137.

De Voogd, B., Truffert, C., Chamot-Rooke, N., Huchon, P., Lallemant, S., Le Pichon, X., 1992. Two-ship deep seismic sounding in the basins of the Eastern Mediterranean Sea (Pasiphae cruise). Geophys. J. Int. 109, 536-552.

Haese R., De Lange G., 2000. Pore water geochemistry of $\mathrm{CH}_{4}$ rich mud volcano sediments in the East Med Sea: Implications for the anaerobic methane oxidation, presence of gas hydrates and intensive irrigation. Goldschmidt 2000 Conference (Oxford, 3-8/09/2000).

Hieke, W., Bizon, G., Müller, C., 1985. Middle Miocene to Quaternary sediments from the Mediterranean Ridge (Ionian Sea) and geodynamic implications. "Meteor" Forschungsergebn C39, 1-22.

Huguen, C., Mascle, J., Chaumillon, E., Kopf, A., Woodside, J., 00. Tectonic control on Mud Volcanoes: Evidences from the Central and Eastern Mediterranean Ridge. Am. Assoc. Petrol. Geol. Bull. (soumis).

Hsü, K.-J., Montardet, L., 1978. Init Report DSDP 42 (1).

Kopf, A., Robertson, A.H.F., Clennel, M.B., Flecker, R., 1998. Mechanisms of mud extrusion on the Mediterranean Ridge Accretionary Complex. Geo-Mar. Lett. 18, 97-114.
Kopf, A., Robertson, A.H.F., Volkmann, N., 2000. Origin of mud breccia from the Mediterranean Ridge accretionary complex based on evidence of the maturity of organic matter and related petrographic and regional tectonic evidence. Mar. Geol. 166, 65-82.

Le Pichon, X., Chamot-Rooke, N., Lallemant, S., 1995. Geodetic determination of the kinematics of central Greece with respect to Europe: Implications for Eastern Mediterranean Tectonics. J. Geophys. Res. 100, 12675-12690.

Limonov, A.F., Woodside, J.M., Cita, M.B., Ivanov, M.K., 1996. The Mediterranean Ridge and related mud diapirism: a background. Mar. Geol. 132, 7-19.

Maltman, A., 1994. The Geological Deformation of Sediments. Chapman \& Hall.

Mascle, J., Huguen, C., Benkhelil, J., Chamot-Rooke, N., Chaumillon, E., Foucher, J.-P., Griboulard, R., Kopf, A., Lamarche, G., Volkonskaia, A., Woodside, J., Zitter, T., 1999. Images may show start of European-African plate collision. Eos Trans. Am. Geophys. Union 80, 421, 425, 428.

Mc Clusky, S., Balassanian, S., Barka, A., Demir, C., Ergintav, S., Georgiev, I., Gurkan, O., Hamburger, M., Hurst, K., Kahle, H., Kastens, K., Kekelidze, G., King, R., Kotzev, V., Lenk, O., Mahmoud, S., Mishin, A., Nadariya, M., Ouzounis, A., Paradissis, D., Peter, Y., Prilepin, M., Reillinger, R., Sanli, I., Seeger, H., Tealeb, A., Toksöz, M.-N., Veis, G., 2000. Global Positioning System constraints on plate kinematics and dynamics in the Eastern Mediterranean and Caucasus. J. Geophys. Res. 105, NO B3, 5695-5719.

Medinaut/MEDINETH Shipboard Scientific Parties, Linking Mediterranean brine pools and mud volcanism, 2000. Eos Trans. Am. Geophys. Union 81, 625, 631-633.

Milkov, A., 2000. Worldwide distribution of submarine mud volcanoes and associated gas hydrate. Mar. Geol. 167, 29-42.

Milkov, A., Vogt, P., Cherkshev, G., Ginsburg, G., Chernova, N., Andriashev, A., 1999. Seafloor terrains of the Haakon Mosby Mud Volcano as surveyed by deep-tow video and still photography. Geo-Mar. Lett. 19, 38-47.

Milliman, J.-D., 1974. Marine Carbonates. Springer-Verlag, New York.

Milliman, J.D., Müller, J., 1973. Precipitation and lithification in the deep-sea of magnesian calcite in the deep-sea sediments of the eastern Mediterranean sea. Sedimentology 20, 29-45.

Rellinger, R.E., McClusty, S.C., Oral, M.B., King, R.W., Toksoz, M.N., 1997. Global Positioning System measurements of present-day crustal movements in the Arabia-Africa-Eurasia plate collision zone. J. Geophys. Res. 102, 9983-9999.

Robertson, A., Shipboard scientific party, 1996. Mud volcanism on the Mediterranean Ridge: Initial results of Ocean Drilling Program Leg 160. Geology 24, 239-242.

Robertson, A.-H.-F., Kopf, A., 1998. Tectonic setting and processes of mud volcanism on the Mediterranean Ridge accretionary complex: evidence from Leg 160, ODP Scientific Results, vol. 160, pp. 569-574.

Vengosh, A., De Lange, G.J., Starinsky, A., 1998. Boron isotopes and geochemical evidence for the origin of Urania and Bannock brines at the eastern Mediterranean: effect of water-rock interaction. Geochim. Cosmochim. Acta 62, 3221-3228. 
Vogt, P.-R., Cherkashev, G., Ginsburg, G., Ivanov, G., Milkov, A., Crane, K., Lein, A., Sundvor, E., Pimenov, N., Egorov, A., 1997. Haakon Mosby mud volcano provides unusual example of venting. Eos Trans. Am. Geophys. Union 78, 549, 556-557.

Vogt, P.R., Gardner, J., Crane, K., 1999. The Norvegian-BarentsSvalbard continental margin: Introducing a natural laboratory. Geo-Mar. Lett. 19, 2-21.

Volgin, A.V., Woodside, J.M., 1996. Sidescan sonar images of mud volcanoes from the Mediterranean Ridge: possible causes of variations in backscatter intensity. Mar. Geol. 132, 39-53.
Westbrook, G.K., Smith, M.J., 1983. Long decollements and mud volcanoes: Evidence from the Barbados Ridge Complex for the role of high pore-fluid pressure in the development of accretionary complex. Geology 11, 279-283.

Woodside, J.-M., Ivanov, M.-K., Limonov, A.-F., Shipboard scientists of the Anaxiprobe expeditions, 1998. Shallow gaz and gaz hydrates in the Anaximander Mountains region, eastern Mediterranean Sea. In : Henriet, J.-P., Mienert, J. (Eds.), Gas Hydrates: Relevance to World Margin Stability and Climate Change. Geological Society, London, Special Publications 137, pp. 177-193. 